and without the baffle interposed in the spectrometer aperture. The ratio of the two yields was 23.1 , which, multiplied by the solid angle of the aperture, gives a solid angle of $2.52 \times 10^{-3}$ steradian for the spectrometer.

\section{ACKNOWLEDGMENTS}

We wish to acknowledge the support of Watertown Arsenal Laboratory, for whom this work was performed as a part of the development of the application of elastic proton scattering to the analysis of the composition of metal surfaces. Several members of the Institute staff contributed to the design and construction of this apparatus, in particular, Mr. C. I. Glassbrook, who was very helpful in the planning and development of the regulated current supply, and Dr. H. R. Fechter, who designed and installed the flip-coil fluxmeter.

\title{
Operation of Proportional Counters at High Temperatures
}

\author{
A. Moljk, ${ }^{*}$ R. W. P. Drever, and S. C. Curran $\dagger$ \\ Department of Natural Philosophy, Glasgow University, Glasgow, Scotland
}

(Received August 1, 1955)

\begin{abstract}
The operation of proportional counters at temperatures up to $900^{\circ} \mathrm{C}$ has been investigated and it is shown that the factors determining the maximum working temperature are thermal emission of electrons from the cathode, and the conductivity of heated insulating materials. Several types of proportional tubes are discussed and the advantages of counters with external cathodes are pointed out. Pulse spectra from quartz and glass counters containing $K$-capture $\mathrm{A}^{37}$ sources indicate satisfactory operation at temperatures of $810^{\circ} \mathrm{C}$ in the case of quartz and $450^{\circ} \mathrm{C}$ in the case of glass.
\end{abstract}

$\mathrm{T}$ HE proportional tube has proved a very useful instrument in the field of low-energy beta and gamma spectroscopy. ${ }^{1}$ For the energy region below $20 \mathrm{kev}$ in particular, the use of gaseous sources has enabled the errors due to self-absorption and backscattering, which occur in other types of spectrometer, to be eliminated. Corrections are still required for end and wall effects, but these can be made small, and in most cases can be calculated.

Relatively few of the elements can be obtained in suitable gaseous form at room temperature. However, many of them have compounds which show appreciable vapor pressure at somewhat higher temperatures, and could probably be used as constituent gases in a heated proportional counter. Thus, in order to extend the gaseous source technique the operation of proportional counters at high temperatures has been studied.

Previous work on heated counters has been limited to Geiger tubes. ${ }^{2,3}$ A rapid increase of background with temperature has been found and at $200^{\circ} \mathrm{C}$, the highest temperature used, background counting rates of several thousand counts per minute have been reported. ${ }^{4}$

\section{GENERAL CONSIDERATIONS}

The operation of a proportional counter containing a quenching gas depends on the ionization processes

\footnotetext{
* On leave of absence from the University of Ljubljana, Yugoslavia.

$\dagger$ Now at U. K. Atomic Energy Authority, Aldermaston, Berkshire, England.

'See S. C. Curran in Beta and Gamma-Ray Spectroscopy edited by K. Siegbahn (North-Holland Publishing Company, Amsterdam, 1955).

${ }^{2}$ C. P. Joshi, Indian J. Phys. 27, 393 (1953).

${ }^{3}$ Om Parkash, Phys. Rev. 80,303 (1950).

4 Meunier, Bonpas, and Legrand, J. phys. radium 16, 145 (1955).
}

taking place along the track of the primary particle and in the avalanches where the secondary electrons are multiplied. The energies required in these processes are large compared with the average thermal energy of the gas molecules which, even at $1000^{\circ} \mathrm{C}$, are less than $0.2 \mathrm{ev}$. Thus it might be expected that an increase in the temperature of a closed counter, in which the mean free path remains constant, would not affect its operation directly.

However, a limit to the working temperature is set by any thermal process which produces electrons or negative ions in the counter, such as thermal emission, or the ionization of gas molecules on hot surfaces. The temperature at which thermionic emission becomes important cannot be accurately predicted as the presence of gas (and impurities generally) may alter the effective work function appreciably. In vacuum a cathode of copper with a thermionic work function of $4.3 \mathrm{ev}$ would emit 1 electron per $\mathrm{cm}^{2}$ per second at a temperature of $540^{\circ} \mathrm{C}$ and $10^{6}$ electrons per $\mathrm{cm}^{2}$ per second at $770^{\circ} \mathrm{C}$. In a proportional counter the space charge accompanying the latter counting rate would certainly prevent normal operation.

\section{DESIGN OF COUNTERS}

A serious difficulty in the construction of a counter for high temperature is the low electrical resistivity of heated insulating materials. Borosilicate glass, for instance, has at room temperature a resistivity of the order of $10^{14} \mathrm{ohm} \mathrm{cm}$ which falls to $10^{7} \mathrm{ohm} \mathrm{cm}$ at $400^{\circ} \mathrm{C}$, while the resistivity of fused quartz, one of the best insulating materials, reaches the latter value at $700^{\circ} \mathrm{C}$

Insulation difficulties may be avoided by keeping 


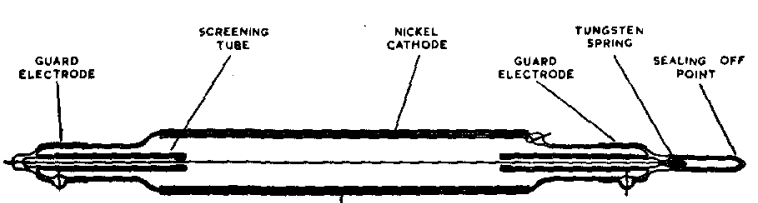

FrG. 1. Proportional counter of hard glass for use at temperatures up to $450^{\circ} \mathrm{C}$. Thickened lines indicate conducting coatings on the glass.

the insulators cold and this method was used in some of the preliminary experiments. However, a counter of this type is not suitable for vaporized sources which would condense on the cold surfaces.

For temperatures up to $450^{\circ} \mathrm{C}$ a hard glass counter such as shown in Fig. 1 can be used. It differs from conventional design in having a considerable length of glass insulation at both ends of the cathode. In addition wide rings of graphite on the surface of the glass act as guard electrodes, preventing the large leakage current reaching the wire. The cathode is of nickel, which has a work function of $5.0 \mathrm{ev}$.

Proportional counters with external cathodes can also be used. $^{5}$ A quartz counter of this type which operates satisfactorily at temperatures up to $800^{\circ} \mathrm{C}$, is shown in Fig. 2. The tungsten wire, of diameter 0.1 $\mathrm{mm}$, is spot-welded to the molybdenum strip seal and is kept tight by a tungsten spring. The cathode and guard rings are films of graphite, or evaporated metal, on the outside surface of the quartz.

At temperatures above $180^{\circ} \mathrm{C}$ the quartz is sufficiently conducting to prevent charges accumulating on the inside surface, and the counter operates as if it had a normal internal cathode. However at room temperature when the quartz is a good insulator the counter behaves as if a condenser were connected in series with the cathode. During counting charge collects on this condenser, causing a slow decrease in gain if the applied voltage is constant. In addition changes of the sensitive volume occur due to variations in the distribution of charge on the quartz surface between the cathode and the guard rings. Quartz counters with external cathodes are therefore unsuitable for accurate work at temperatures below $180^{\circ} \mathrm{C}$ but for higher temperatures they have several advantages. They can be made relatively easily and chemical decomposition of filling gases is less likely in the absence of large metal surfaces.

Glass counters of similar design are also convenient for many purposes, and operate at temperatures between $120^{\circ} \mathrm{C}$ and $450^{\circ} \mathrm{C}$ when borosilicate glass is used.

\section{EXPERIMENTAL TECHNIQUE}

In a comprehensive check of the operation of a proportional counter energy resolution has to be measured, and the absence of spurious pulses must be established. A measurement of the dependence of

${ }^{5}$ A. L. Cockcroft and J. M. Valentine, J. Sci. Instr. 27, 262 (1950). counting rate on the voltage applied to the counter gives some information about both of these factors, and this method was widely used before multichannel pulse analyzers were available. A more sensitive test is the analysis of the pulse-height distribution obtained when short-range monoenergetic electrons are produced throughout the sensitive volume of the counter.

In practice a beam of monoenergetic $x$-rays can be fired into the counter, or a suitable gaseous $K$-capture source can be included in the filling. The latter method was employed in the present experiments, using the $K$ and $L$ radiations from $\mathrm{A}^{37}$ sources.

A standard technique was developed for preparing and testing the various counters. Each counter was first carefully degassed for several hours under a vacuum better than $10^{-5} \mathrm{~mm}$ of mercury at a temperature of $1000^{\circ} \mathrm{C}$, when made from quartz, and $540^{\circ} \mathrm{C}$ when of glass. The counter was then filled with argon and carbon dioxide to partial pressures of $30 \mathrm{~cm}$ and 7 $\mathrm{cm}$ of mercury respectively, together with a trace of $\mathrm{A}^{37}$, and sealed off. The total gas pressure was chosen to prevent excessive pressure when the counter is hot. The counter was arranged in a thermostatically controlled furnace, screened electrically by stainless steel sheet. The high voltage connection was made through a quartz tube, while the signal was brought out through a screened lead with ceramic insulation. The pulses from the counter, after amplification, were examined on an oscilloscope and analyzed by a Hutchinson-Scarrott 100-channel kicksorter. For temperature measurement a platinum-platinum rhodium thermocouple was used. Pulse spectra and counting rates were measured at various temperatures, with gas multiplication factors ranging from 2000 to 20000 .

\section{GLASS COUNTERS}

Glass counters both with internal and external cathodes have operated satisfactorily at temperatures up to $520^{\circ} \mathrm{C}$. Pulse distribution spectra and counting rates taken with the same applied voltages at various temperatures between $120^{\circ} \mathrm{C}$ and $520^{\circ} \mathrm{C}$ were identical within statistical errors. Typical spectra taken at $450^{\circ} \mathrm{C}$ in a counter with a nickel cathode are given in Fig. 3, which shows the $2.8 \mathrm{kev} \mathrm{A}^{37} K$-peak, and in Fig. 4, which shows the 240 ev $L$ peak. Such curves indicated that the operation of the counter down to energies of $70 \mathrm{ev}$ was completely unaffected by change of temperature within the range stated. It may be remarked that the dimensions of the counter were limited by the furnace available, so end effects were

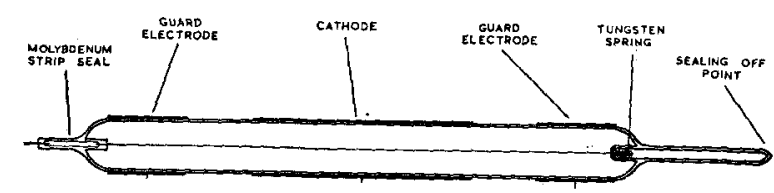

FIG. 2. External cathode counter of fused quartz for operation at temperatures up to $800^{\circ} \mathrm{C}$. 


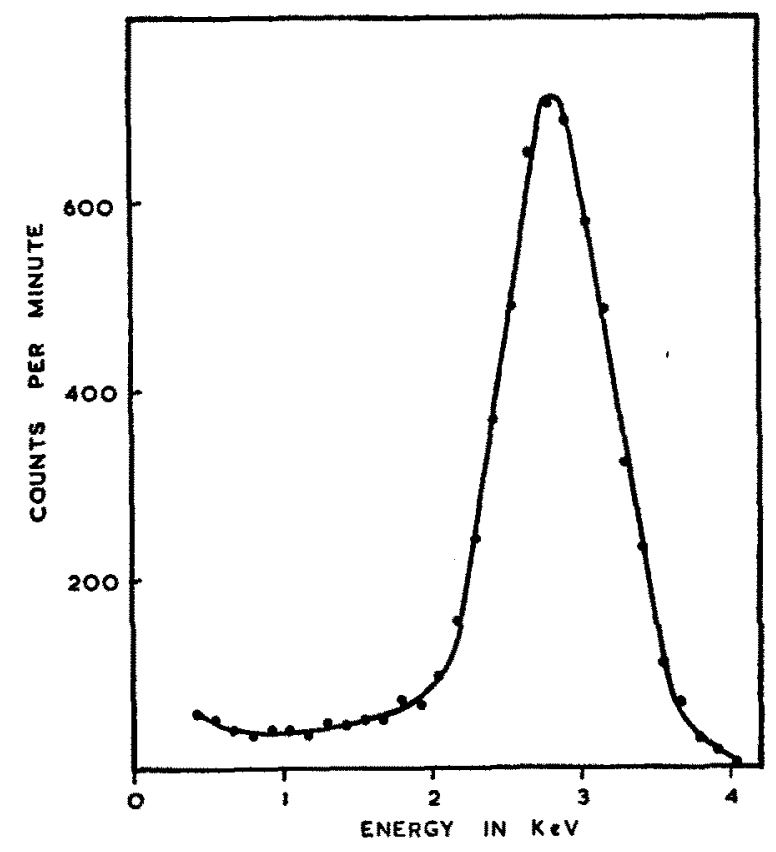

Fig. 3. $K$-peak of $A^{37}$ taken in a glass counter at $450^{\circ} \mathrm{C}$.

relatively large. Better energy resolution can be obtained with longer counters.

The factor limiting the operating temperature of the glass counters was the electrical conductivity of the glass. In the present experiments a borosilicate glass ${ }^{6}$ was used. At $540^{\circ} \mathrm{C}$, the maximum working temperature, the current flowing through the glass from the cathode to the guard rings was $4 \mathrm{ma}$, while at $425^{\circ} \mathrm{C}$ it was $320 \mu \mathrm{a}$, and at $330^{\circ} \mathrm{C} 20 \mu \mathrm{a}$.

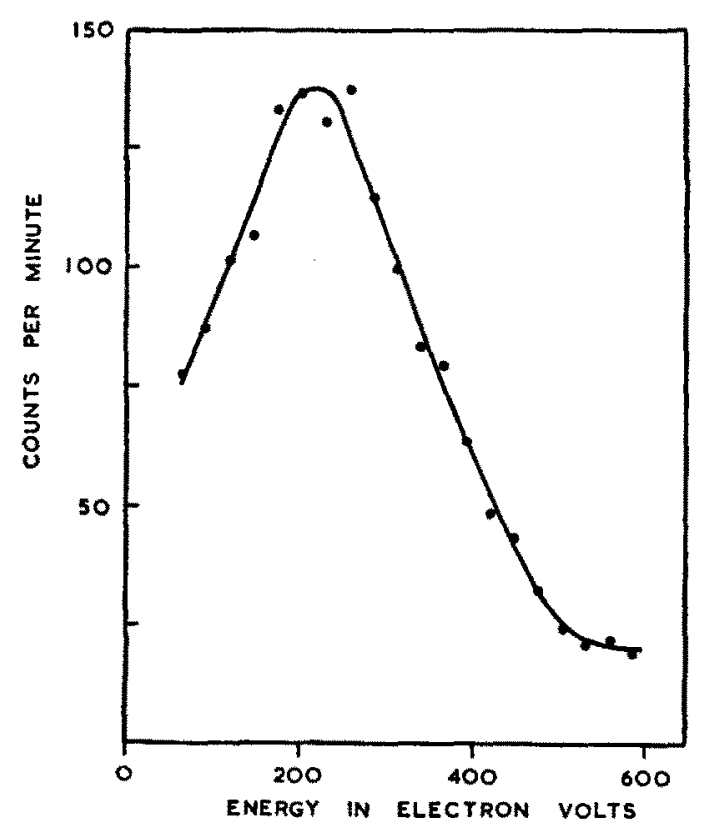

Fig. 4. L-peak of $A^{37}$ taken in a glass counter at $450^{\circ} \mathrm{C}$.

- C9 by British Thomson-Houston Company Ltd.
When glass counters were operated with appreciable leakage current, electrolysis effects appeared. The graphite films on the surface of the glass became less efficient as guard rings and after about one coulomb of electricity had passed, direct leakage to the wire occurred. This effect is due to removal of metallic ions from the vicinity of the guard rings with a resultant increase of resistivity there. The graphite electrodes thus become effectively insulated from the glass. At high temperatures this limits the life of the type of glass counter described, but this difficulty could be avoided by using metal guard rings sealed to the glass.

Another effect related to the decrease of resistivity of glass with temperature was overshoot, which became apparent at about $400^{\circ} \mathrm{C}$. This was caused by a reduction of the time constant of the input circuit to a value comparable with the differentiation time constant, $15 \mu \mathrm{sec}$, in the amplifier.

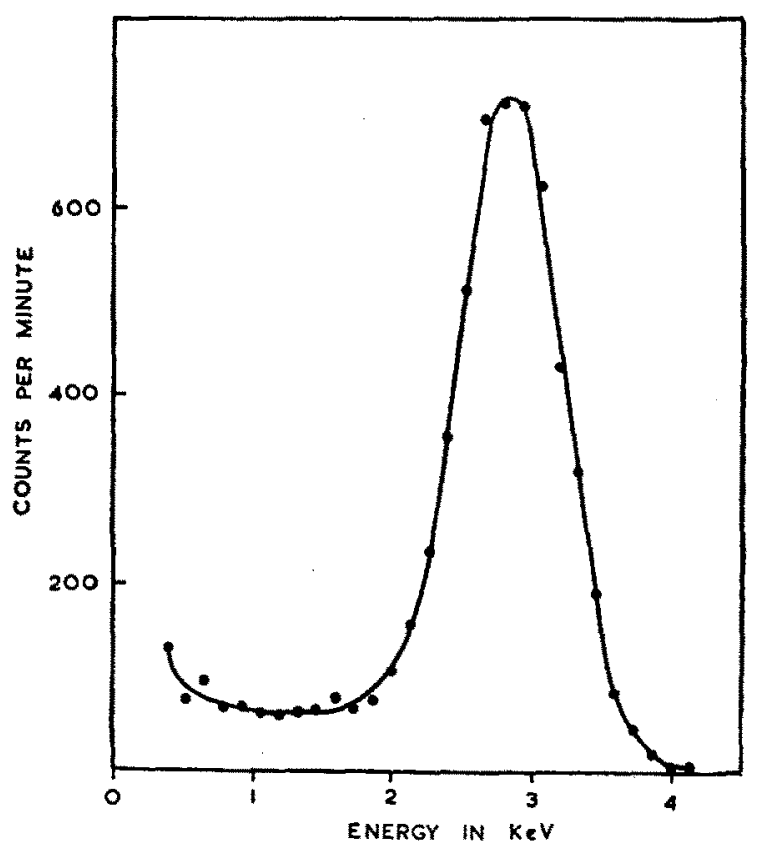

Fic. 5. $K$-peak of $A^{37}$ taken in a quartz counter at $810^{\circ} \mathrm{C}$.

In the first experiment the head amplifier was directly connected to the counter wire, and at temperatures above $400^{\circ} \mathrm{C}$, a reduction in the anode current of the input valve was noted. Further work showed that a cell formed by the graphite and tungsten electrodes with the glass as electrolyte was generating an emf of 0.5 volt. In later experiments an external voltage source was used to compensate for this, or condenser coupling was employed.

A spurious effect observed in a counter with an internal nickel cathode is worth noting. At about $170^{\circ} \mathrm{C}$ spurious pulses appeared and rapidly became more frequent with further increase in temperature. The pulses were due to sparking between the cathode and the slightly conducting glass. This effect was prevented 
by coating the outside of the glass with graphite, as shown in Fig. 1, and connecting to the cathode. The conductivity of heated glass seems to have been overlooked in previous work with Geiger counters and spurious effects of this kind may be one of the reasons for the reported great increase of background with temperature.

\section{QUARTZ COUNTERS}

With fused quartz conductivity does not become appreciable until much higher temperatures, and in the counter shown in Fig. 2 a leakage current to the guard rings of $4 \mathrm{ma}$ was not reached until $800^{\circ} \mathrm{C}$. Such a counter can thus be used at higher temperatures than a glass one, and Fig. 5 shows a $2.8 \mathrm{kev} \mathrm{A}{ }^{37}$ peak obtained at $810^{\circ} \mathrm{C}$ with this counter.

In the present experiments with quartz counters, however, the conductivity of quartz was not the only factor limiting the operating temperature. Above $770^{\circ} \mathrm{C}$ small pulses corresponding to primary particle energies of less than $80 \mathrm{ev}$ became apparent on the oscilloscope. With further increase of temperature these pulses rapidly became more numerous, and piling up occurred. On the oscilloscope this had the appearance of noise. There was also a slight decrease in counter gain due to space charge effects. At $870^{\circ} \mathrm{C}$, although the $2.8 \mathrm{kev}$ $\mathrm{A}^{37}$ peak was still clearly visible, that part of the primary particle spectrum below $1 \mathrm{kev}$ was hidden by the noise. The ratio between the $2.8 \mathrm{kev}$ pulses and the amplitude of the noise was independent of counter gain, so the spurious effect was evidently due to electrons or ions produced in the sensitive volume and multiplied. The phenomenon can, in fact, be explained as a piling up of small pulses due to thermal emission from the cathode. A very approximate estimation of the work function from these experiments suggests a value which is in agreement with the published value for silica of $4.8 \mathrm{ev}$.

In a counter with an internal cathode coated by carbon, exactly similar effects were observed at temperatures between $500^{\circ} \mathrm{C}$ and $680^{\circ} \mathrm{C}$. This gives further support to the explanation above since appreciable thermal emission would be expected at these peratures from carbon, whose work function is $4.4 \mathrm{ev}$.

\section{ACKNOWLEDGMENTS}

One of us (R.W.P.D.) would like to thank the Department of Scientific and Industrial Research for a grant, during tenure of which the work was performed

\title{
Helium-3 Filled Proportional Counter for Neutron Spectroscopy
}

\author{
R. Batchelor, R. Aves, and T. H. R. Skyrme: \\ Atomic Energy Research Establishment, Harwell, Berkshire, England
}

(Received August 4, 1955)

\begin{abstract}
The neutron transformation of helium-3 has been applied to neutron spectroscopy in the medium energy range in order to overcome the disadvantages of previous methods which have usually been based on neutron scattering. By observing the pulse-height distribution when a proportional counter filled with helium-3, krypton, and carbon dioxide is irradiated with neutrons, the spectrum can be obtained. Details of the counter construction and vacuum equipment for filling the counter are described. In the range of energy from thermal to $1 \mathrm{Mev}$ the spread (relative standard deviation of a peak) is about $3 \%$ and the wall effect agrees with theoretical predictions. The pulse height varies linearly with energy and some evidence suggesting that the mean energy required to produce an ion pair in krypton for helium-3 nuclei is about $6 \%$ greater than that for protons is presented. Since the neutron reaction cross section of helium-3, as a function of energy, is required to evaluate neutron spectra from observed distributions, it has been remeasured in the range $120 \mathrm{kev}$ to $1 \mathrm{Mev}$ with greater accuracy than previous measurements made by J. H. Coon [Phys. Rev. 80, $448(1950)]$.
\end{abstract}

\section{INTRODUCTION}

$T^{\mathrm{H}}$ HE use of a proportional counter filled with helium- 3 as a device for measuring fast neutron spectra has been previously reported. ${ }^{1}$ The preliminary work outlined there was carried out with a small quantity of helium with the helium-3 content enriched to $10 \%$. More recently Dr. E. Bretscher, who originally suggested the development of this instrument, has acquired on loan from the U.S.A. a liter of pure helium-3. This communication describes in detail a more highly developed spectrometer which makes use

\footnotetext{
${ }^{1}$ R. Batchelor, Proc. Phys. Soc. (London) A65, 674 (1952).
}

of some of this gas, and also the vacuum system required for filling the counter. It will be appreciated that in view of the high cost of helium -3 a considerable amount of technical development was involved. A reliable pressure-vacuum valve, gas recovery system, and other items described in Sec. 3 had to be designed. Details of one application, the measurement of the intensity of the second group of neutrons from the proton bombardment of lithium-7, have been published. ${ }^{2,3}$

${ }^{2}$ R. Batchelor, Proc. Phys. Soc. (London) A68, 452 (1955).

${ }^{3}$ R. Batchelor and G. C. Morrison (to be published). 